\title{
Frontal lobe syndrome caused by a giant meningioma presenting as depression and bipolar disorder
}

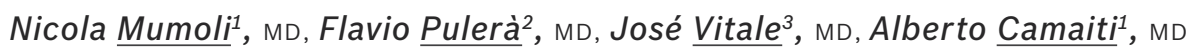

ABSTRACT Frontal meningiomas may present only with psychological symptoms that resemble depression, anxiety states, hypomania and schizophrenia. Herein, we present the case of a 55-year-old man who was initially thought to have depression and bipolar disorder, but was eventually diagnosed with frontal lobe syndrome caused by a giant frontal meningioma.

Keywords: behavioural abnormalities, bipolar disorder, depression, frontal lobe syndrome, meningioma

\section{INTRODUCTION}

Meningiomas are the most common benign brain tumours, accounting for $13 \%-26 \%$ of intracranial tumours. ${ }^{(1)}$ The reported incidence is low because most meningiomas are asymptomatic. Meningiomas that compress the frontal lobes are notoriously 'silent', and are thus likely to be misdiagnosed or overlooked. Patients with such tumours are often referred first to psychiatrists because they may present with psychological symptoms resembling depression, anxiety states, hypomania, and schizophrenia.

\section{CASE REPORT}

A 55-year-old man with a history of depression, behavioural abnormalities and alcohol abuse was admitted in February 2010 for vomiting and confusion. He had been well, but began to express strange and unusual thoughts two years prior to his admission. Before he was observed to have such symptoms, the patient's medical and family history were unremarkable, and he was not on any medications or illicit drugs and had never smoked or consumed alcohol. His wife noted that his change in behaviour had been gradual, with the patient becoming more distracted, euphoric and disinhibited with time. He subsequently experienced fatigue, anxious restlessness and hypersensitivity to external noises. He also started to consume alcohol excessively. Despite receiving a prescription for anxiolytics and paroxetine from a local psychiatrist, his symptoms worsened, and in April 2009, the patient was dismissed from his job due to poor performance and absenteeism. When intoxicated with alcohol, the patient presented with agitation, irritability and combativeness, and had once hit his wife - something he had never previously done. At that time, the psychiatric diagnosis was bipolar disorder and alcoholic abuse, for which oral risperidone was prescribed. However, there was only a mild and temporary improvement in the patient's condition. In October 2009,
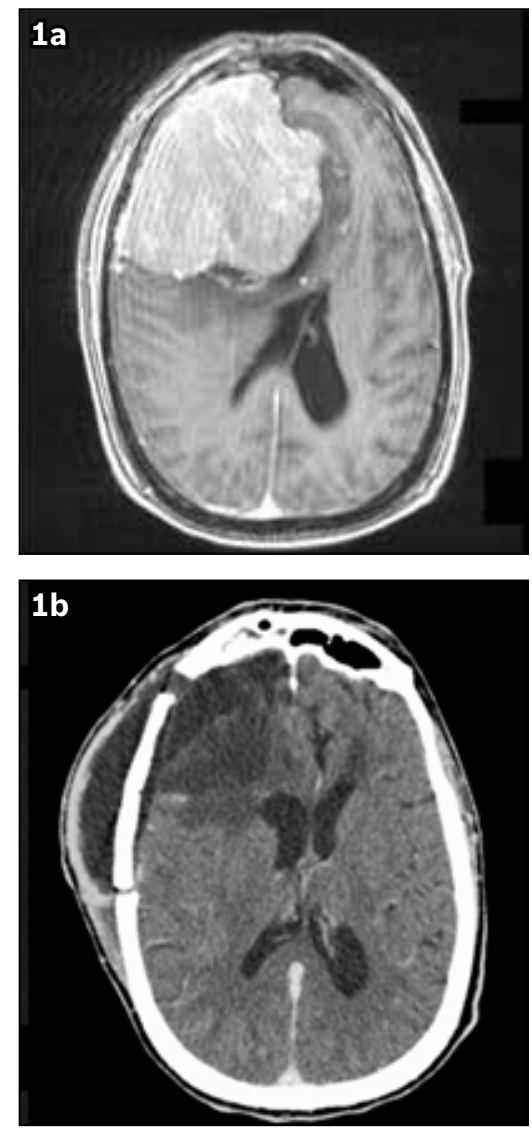

Fig. 1 (a) MR image of the brain shows a $7.5 \mathrm{~cm} \times 7.4 \mathrm{~cm}$ mass involving the right frontal area, suggestive of a giant meningioma. (b) CT image of the brain shows complete removal of the tumour.

his wife left him, and he relocated to a country house, living in unhygienic conditions and refusing any external help.

On arrival at our hospital, the patient was euphoric and in a state of confusion. He also had reduced concentration and disinhibited thoughts. The patient had poor self-care and hygiene, and wore the same clothes every day. He showed no concern over his appearance, and his ability to communicate was very poor. He weighed $50 \mathrm{~kg}$ and his body mass index

${ }^{1}$ Department of Internal Medicine, ${ }^{2}$ Department of Neurosurgery, Ospedale Civile Livorno, ${ }^{3}$ Department of Internal Medicine, Ospedale di Circolo, Varese, Italy Correspondence: Dr Nicola Mumoli, Chief of Section for Vascular Medicine, Department of Internal Medicine, Ospedale Civile Livorno, Viale Alfieri 36 , 57100 Livorno, Italy. nimumoli@tiscali.it 
was $18 \mathrm{~kg} / \mathrm{m}^{2}$. Blood test results were normal, except for mild macrocytic anaemia and slightly high transaminases, gamma-glutamyl transferase and blood-alcohol concentration. Psychiatric consultation confirmed bipolar disorder with acute alcoholic toxicity, and sodium valproate was added to his previous therapy.

Neurological examination was unremarkable apart from a swollen right optic disk, which was observed on funduscopic examination. Subsequent brain magnetic resonance (MR) imaging revealed an unexpected mass measuring $7.5 \mathrm{~cm} \times$ $7.4 \mathrm{~cm}$ at the right frontal area, suggestive of a giant meningioma (Fig. 1a). Accordingly, a diagnosis of frontal lobe syndrome was made, and the patient was transferred to the neurosurgery department, where he underwent a right frontal craniotomy with uneventful resection of the meningioma (Fig. 1b). After the patient was discharged, he was given regular group and individual psychotherapy. He was also provided with psychiatric follow-up and home help for physiotherapy and nutrition monitoring. Repeat brain MR imaging performed after ten months of follow-up showed no recurrence of the tumour; all psychiatric symptoms had resolved and the patient had stopped drinking alcohol. He also had a weight gain of $8 \mathrm{~kg}$. When last seen in June 2011, the patient was able to care for himself with the support of a social worker. He had resumed work and reunited with his wife.

\section{DISCUSSION}

The patient's former history and fluctuant symptoms with alcohol-related complications were prioritised in our differential diagnosis. Wernicke-Korsakoff syndrome is well associated with the development of the aforementioned clinical manifestations. The classic triad of symptoms (eye signs, ataxia and confusion) do not always present together, with many patients presenting with only one symptom, most commonly mental change, which was the symptom observed in our patient. ${ }^{(2)}$

Primitive frontal lobe tumours tend to be misdiagnosed or overlooked in patients with a history of alcohol abuse, depression or personality changes, as the clinical features may overlap. Diagnosis is often delayed due to the insidious nature of the symptoms, which may be mistaken as symptoms of depression or psychotic syndromes like hypomania and schizophrenia. Furthermore, after a patient has been diagnosed to have a psychiatric disorder, the case is rarely reviewed for possible organic causes.

The best known report of frontal lobe dysfunction involves Phineas Gage, a railroad construction foreman, who became famous for his profound personality change after a brain injury that destroyed most of his brain's frontal lobe. ${ }^{(3)}$ The case reported here represents a typical illustration of 'executive dysfunction syndrome', which is now preferentially termed 'frontal lobe syndrome' because impairment of executive functions have also been observed in patients without frontal lesions. Although the term 'frontal lobe syndrome' is not found in current textbooks or articles and lacks a widely accepted definition, it continues to be used in the clinical setting.(4) Causes of frontal lobe syndrome include cerebrovascular disease, head trauma, brain tumours, brain infections and neurodegenerative diseases.

Meningiomas are the most common benign brain tumour, accounting for $13 \%-26 \%$ of intracranial tumours. ${ }^{(1)}$ However, the reported incidence is low because many meningiomas do not present with symptoms. Frontal meningiomas may present with only psychological symptoms that resemble depression, anxiety states, hypomania and schizophrenia. ${ }^{(5)}$ In Gupta and Kumar's study of 79 meningiomas, 15 (21\%) patients presented with psychiatric symptoms in the absence of neurological symptoms. ${ }^{(6)}$ While it is not practical for a psychiatrist to subject every patient who presents with psychological problems to a neuroradiological investigation, there are features that present in the early stages of psychological disorders that are indicative of a serious organic disease. These features include no previous history of psychiatric disorder, the onset of symptoms after 35 years of age and a slowly progressive psychological change - all of which increase the likelihood of a diagnosis of frontal lobe syndrome. Patients who present with these features should be screened using computed tomography or MR imaging to detect possible intracranial lesions (e.g. tumours, subdural haematomas and hydrocephalus) or diseases (e.g. cerebrovascular disease) that may cause or contribute to frontal lobe syndrome. ${ }^{(6)}$

In conclusion, primitive frontal lobe tumours are likely to be misdiagnosed or overlooked. Patients with such tumours are often referred first to psychiatrists. When a young person with no history of psychiatric disease develops a slow and progressive psychological change, frontal lobe syndrome should be considered. Papilloedema may develop only when the meningioma has reached an advanced stage.

\section{REFERENCES}

1. Whittle IR, Smith C, Navoo P, Collie D. Meningiomas. Lancet 2004; 363:1535-43.

2. Thomson AD, Cook CC, Guerrini I, et al. Wernicke's encephalopathy: 'Plus ca change, plus c'est la meme chose'. Alcohol Alcohol 2008; 43:180-6.

3. Harlow JM. Passage of an iron rod through the head. Boston Med Surg J 1848; 39:389-93

4. Godefroy O. Frontal syndrome and disorders of executive functions. J Neurol 2003; 250:1-6.

5. Maurice-Williams RS, Dunwoody G. Late diagnosis of frontal meningiomas presenting with psychiatric symptoms. Br Med J 1988; 296:1785-6.

6. Gupta RK, Kumar R. Benign brain tumours and psychiatric morbidity: a 5-years retrospective data analysis. Aust N Z J Psychiatry 2004; 38:316-9. 